\title{
Electrochemical studies on small electron transfer proteins using membrane electrodes
}

\author{
M.M. Correia dos Santos ${ }^{\text {a,* }}$, P.M. Paes de Sousa ${ }^{\text {a }}$, M.L. Simões Gonçalves ${ }^{\text {a }}$, \\ L. Krippahl ${ }^{\mathrm{b}}$, J.J.G. Moura ${ }^{\mathrm{b}}$, E. Lojou ${ }^{\mathrm{c}}$, P. Bianco ${ }^{\mathrm{c}}$ \\ ${ }^{a}$ Centro de Química Estrutural, Instituto Superior Técnico, Av. Rovisco Pais 1, 1049-001 Lisbon, Portugal \\ ${ }^{\mathrm{b}}$ Departamento de Quimica, REQUIMTE, Centro de Quimica Fina e Biotecnologia, Faculdade de Ciências e Tecnologia, Universidade Nova de Lisboa, \\ 2825-114 Monte da Caparica, Portugal \\ ${ }^{\mathrm{c}}$ Unité de Bioénergétique et Ingénierie des Protéines, CNRS, 31 Chemin Joseph Aiguier, F-13402 Marseille Cedex 20, France
}

Received 4 July 2002; received in revised form 6 November 2002; accepted 18 November 2002

\begin{abstract}
Membrane electrodes (ME) were constructed using gold, glassy carbon and pyrolytic graphite supports and a dialysis membrane, and used to study the electrochemical behavior of small size electron transfer proteins: monohemic cytochrome $c_{522}$ from Pseudomonas nautica and cytochrome $c_{533}$ as well as rubredoxin from Desulfovibrio vulgaris. Different electrochemical techniques were used including cyclic voltammetry $(\mathrm{CV})$, square wave voltammetry (SW) and differential pulse voltammetry (DP). A direct electrochemical response was obtained in all cases except with rubredoxin where a facilitator was added to the protein solution entrapped between the membrane and the electrode surface. Formal potentials and heterogeneous charge transfer rate constants were determined from the voltammetric data. The influence of the ionic strength and the $\mathrm{pH}$ of the medium on the electrochemical response at the ME were analyzed. The benefits from the use of the ME in protein electrochemistry and its role in modulating the redox behavior are analyzed. A critical comparison is presented with data obtained at non-MEs. Finally, the interactions that must be established between the proteins and the electrode surfaces are discussed, thereby modeling molecular interactions that occur in biological systems.
\end{abstract}

(C) 2002 Elsevier Science B.V. All rights reserved.

Keywords: Membrane electrodes; Cytochromes $c_{552}$ and $c_{553}$; Rubredoxin; Unmediated electrochemistry

\section{Introduction}

It is now well recognized that electrochemical techniques are powerful tools for characterizing electrontransfer properties of proteins and catalysis by enzymes. Since the pioneer work of Yeh and Kuwana [1] that reported the direct electrochemistry of cytochrome $c$ at an oxide electrode, and that of Eddowes and Hill [2], which found that essentially reversible voltammetry of the same protein could be observed at a 4,4'-dipyridyl modified gold electrode, significant advances have been made in unmediated electrochemistry of redox proteins.

\footnotetext{
* Corresponding author. Tel.: +351-21-841-9272; fax: +351-21846-4455.

E-mail address: mcsantos@alfa.ist.utl.pt (M.M. Correia dos
} Santos).
The direct electrochemistry of redox proteins can be modulated by several factors and over the years much has been written about all the aspects that can lead to and/or influence the charge transfer [3-6]. Special attention has been focused on the nature of the electrode and electrode $\mid$ solution interface, adsorption phenomena of the proteins at the electrode surfaces and the role of hydrogen-bonding capabilities as well as electrostatic interactions between the protein molecules and the electrode surface. Consequently, a wide range of working electrodes and strategies has been applied to the study of direct electrode processes.

Metal electrodes, like gold, have been considered very poor as interfaces for direct electron transfer unless a suitable compound modifies, them allowing favorable and reversible binding of the redox protein. The use of a facilitator further prevents an irreversible adsorption of 
the protein at the electrode surface that can cause denaturation processes. It has been claimed that bare electrodes are more prone to be affected by the presence of impurities, as well as by the previous treatment of the electrode surface. The presence of a modifier can be a way to circumvent the lack of reproducibility in the voltammetric behavior $[3,7,8]$. So, modified surfaces have been engineered by the use of suitable facilitators. These should contain a surface active group which will bind-adsorb to the electrode surface, as well as groups that may interact with some part of the protein and in this way encourage electron transfer to occur $[9,10]$. Compounds containing nitrogen, sulfur and phosphorous atoms, which may interact with the electrode surface, have been used. Groups that can bind to the protein must be oriented towards the solution and are chosen so that a negative or positive charge is present: the former 'attracts' positively charged proteins and the latter negatively charged proteins. As an example, very poor, or an absence of responses have been found for $c$ type cytochromes at gold electrodes unless in the presence of a suitable modifier. This had been reported in the work of Eddowes and Hill for cytochrome $c$ reduction and many other examples soon followed, for instance, that of the reduction of Desulfovibrio vulgaris cytochrome $c_{553}$ at a gold electrode where no electrochemical response was obtained unless in the presence of 4,4'-bipyridine [11].

Carbon electrodes possess a highly functionalized surface and a net negative charge for $\mathrm{pH}>5.6$ [12]. In this case, well-behaved electrochemistry of redox proteins with the appropriate positive charge has been observed in the absence of an added modifier [13]. The organic-like functionalities on the electrode surface seem to provide a friendly environment for protein interaction with the electrochemical surface. These surfaces can act as spontaneously modified electrodes depending on the degree of functionalities, on the $\mathrm{pH}$ and the previous treatment of the carbon used. For instance, a polished edge plane surface possesses many more carbon oxide functionalities than a basal surface. Accordingly, good voltammetric responses with cationic proteins like cytochrome $c$ have been reported at the edge plane electrode while very poor responses are obtained at the basal electrode $[14,12]$. In the case of negatively charged proteins, promotion of electron transfer has been successfully achieved in the presence of positively charged species [12,15-17].

In any case some type of interaction must take place between the bare or modified electrode surface and the protein [18]. Models were developed, where it has been proposed that electron transfer occurs at specific sites on the electrode $[19,20]$. The electroactive sites may be provided by the electrode surface itself or induced by the modifier.
In spite of the fact that several factors can modulate the electrochemical behavior of redox proteins, previous electrochemical studies on $c$-type cytochromes [21] and on rubredoxin [22], under a variety of experimental conditions, led to sets of results of formal potentials, heterogeneous charge transfer rates and diffusion coefficients that do not differ significantly within the experimental errors. Critical comparison with other published data also revealed a good agreement between all values $[[21,22]$ and references therein]. This prompted us to revisited the electrochemistry of these redox proteins using a new strategy recently proposed that consists of the use of membrane electrodes (ME) with the protein imprisoned between a dialysis membrane and the electrode surface [23-25]. Several important advantages can be pointed out: (i) only very small volumes $(2 \mu \mathrm{l})$ of protein are necessary (in several cases a major obstacle can be the very small amounts of protein sample available for investigations); (ii) ease of electrode preparation and low cost; (iii) the capacity to investigate various experimental parameters rapidly and also (iv) the thin layer configuration which easily allows the determination of quantitative information about the redox processes. Moreover, since the membranes used for the construction of the ME are charged, a convenient way to modulate the electric environment close to the electrode surface is offered and thus the role of electrostatic interactions in the efficiency of the electron transfer may be ascertained.

In this work glassy carbon, pyrolytic graphite and gold MEs are used to explore the electrochemical response of two $c$-type cytochromes ( $c_{552}$ from Pseudomonas nautica and $c_{533}$ from $D$. vulgaris) and of rubredoxin from $D$. vulgaris. These proteins are all well characterized redox systems with a wide range of isoelectric points, charges, dipolar moments, surface topographies and redox potentials.

\section{Experimental}

Cytochrome $c_{552}$ from $P$. nautica and cytochrome $c_{533}$ and rubredoxin from $D$. vulgaris were isolated and purified as previously described [26-29]. Poly-L-lysine hydrobromide (PLL) ( $M_{\mathrm{r}}$ 19200) was obtained from Sigma. All other chemicals used were of pro-analysis grade and the solutions were made up with deionized water from a Milli-Q water purification system. The supporting electrolyte was in most cases $10 \mathrm{mM}$ Tris chloride buffer ( $\mathrm{pH}$ 7.6).

Protein stock solutions were prepared in Tris chloride buffer ( $\mathrm{pH}$ 7.6). The concentration of the oxidized forms of the proteins were determined spectrophotometrically using the following molar absorptivities: $\varepsilon_{552}=19000$ $\mathrm{M}^{-1} \mathrm{~cm}^{-1}$ for cytochrome $c_{552}$ [28], $\varepsilon_{553}=23400 \mathrm{M}^{-1}$ 
$\mathrm{cm}^{-1}$ for cytochrome $c_{553}[29]$ and $\varepsilon_{492}=6900 \mathrm{M}^{-1}$ $\mathrm{cm}^{-1}$ for rubredoxin [26].

Voltammetric measurements were performed using a potentiostat/galvanostat from ECO-Chimie, Autolab/ PSTAT 10 as the source of applied potential and as a measuring device. The whole system was controlled by a personal computer that was also used in the data analysis, processed by the GPES software package from ECO-Chimie. In cyclic voltammetry $(\mathrm{CV})$ the scan rate, $v$, was varied between 10 and $200 \mathrm{mV} \mathrm{s}^{-1}$. In square wave voltammetry (SW) the square wave amplitude, $E_{\mathrm{sw}}$, was $50 \mathrm{mV}$, the step height, $\Delta E_{\mathrm{sw}}$ was $10 \mathrm{mV}$ while the frequency was varied between 5 and $100 \mathrm{~Hz}$. In differential pulse voltammetry (DP) the pulse amplitude was 50 or $25 \mathrm{mV}$ and the pulse duration, $t_{\mathrm{p}}$, was varied between 25 and $75 \mathrm{~ms}$. In all experiments the potential was varied between an initial value $E_{\mathrm{i}}$ and a final value $E_{\mathrm{f}}$, depending on the redox potential of the protein under study.

The electrochemical cell featured a conventional three-electrode configuration. The reference electrode was silver | silver chloride with potential of $205 \mathrm{mV}$ versus SHE and the auxiliary electrode was a platinum wire. Several working electrodes were used: a gold electrode $(\mathrm{AuE})$ with nominal radius $=0.08 \mathrm{~cm}$ purchased from BAS (ref. MF-2014), a glassy carbon electrode (GCE) also from BAS (ref. MF-2012) with nominal radius $=0.15 \mathrm{~cm}$ and a pyrolytic graphite electrode (PGE) with nominal radius $=0.2 \mathrm{~cm}$, constructed as previously described [24]. The areas of the electrodes were determined from their response in a known concentration of the ferro-ferricyanide couple [21]. They were found to be close to the nominal areas. Prior to each experiment the PGE and GCE were polished by hand with a water+alumina slurry and then washed with deionized water, and the AuE with emery paper.

The MEs were prepared as previously described $[23,24]$. A small volume $(2 \mu \mathrm{l})$ of the protein solution was deposited on a square piece (about twice the diameter of the electrode sensor) of the dialysis membrane (Spectra/Por MWCO 6000-8000, a negatively charged membrane, symbolized by $\mathrm{M}^{-}$), then the polished electrode was pressed against the membrane and a rubber ring was fitted around the electrode body so that the entrapped solution formed a uniform thin layer. In the experiments with rubredoxin, $2 \mu$ of protein $+1.3 \times 10^{-1} \mathrm{mg} \mathrm{ml}^{-1}$ PLL solution were used. The ME was then placed in the three-electrode cell containing the supporting electrolyte. While small ions can diffuse through the membrane, the protein and PLL molecules will stay entrapped in close vicinity to the electrode surface.

The solutions were deaerated for $10-15$ min with Utype nitrogen that had been previously passed through the supporting electrolyte and then saturated with water. All measurements were done in a temperaturecontrolled room at $T=24 \pm 1{ }^{\circ} \mathrm{C}$.

The dipolar moments were estimated in the following way. An electric field exerts a torque on a dipole, and so the electric dipole moment of a system of charges can be defined by the system's response to an electric field, even if the system is not strictly a dipole (a system of two opposite charges of equal magnitude). For a macromolecule a detailed calculation would not be practical because of the complexity and dynamics of such a system. The calculation implemented in Ref. [30] is a simple approximation: assuming that the molecule would rotate around its geometric center if under the influence of an electric field, the contribution of each charge to the total dipole moment can be calculated as the product of the charge value and the position vector relative to the geometric center. Global charges were calculated per monomer. No charges were used for the prosthetic groups.

\section{Results and discussion}

Rubredoxin is a small iron-sulfur protein (ca. $6 \mathrm{kDa}$ ) with a monomeric iron center surrounded by four cysteinyl thiolate groups, giving a tetrahedral geometry around the metal. The isoelectric point of rubredoxin is low $(\mathrm{p} I \approx 3.5)$ and at $\mathrm{pH} 7$ the protein presents an overall negative charge of -11 [26]. Both cytochromes have methionyl and histidinyl axial coordination, like horse heart cytochrome $c$. Cytochrome $c_{552}$ has a molar mass of $11 \mathrm{kDa}$, a neutral $\mathrm{p} I \approx 7$ and an overall charge of +4 at $\mathrm{pH} 7$, while the molar mass, the $\mathrm{p} I$ and the overall charge at $\mathrm{pH} 7$ of cytochrome $c_{553}$ are $9.1 \mathrm{kDa}$, 8.0 and +9 , respectively $[28,29]$. The dipolar moments calculated, as indicated above, are $322 \mathrm{D}$ for $P$. nautica cytochrome $c_{552}, 215 \mathrm{D}$ for $D$. vulgaris cytochrome $c_{533}$ and $52 \mathrm{D}$ for D. vulgaris rubredoxin. Protein Data Base was used in these calculations.

\subsection{Electrochemistry of cytochromes $c_{552}$ and $c_{553}$ at MEs}

The electrochemical behavior of cytochrome $c_{552}$ was analyzed at membrane pyrolytic graphite (MPGE) and membrane glassy carbon (MGCE) electrodes using CV and SW voltammetry. Typical cyclic voltammograms at the MPGE are shown in Fig. 1 for a $100 \mu \mathrm{M}$ cytochrome $c_{552}$ solution entrapped between the membrane and the electrode surface. In Fig. 2, square wave voltammograms for a $100 \mu \mathrm{M}$ cytochrome $c_{522}$ solution at the MGCE are shown. In both situations the ME was immersed in $10 \mathrm{mM}$ Tris chloride buffer solution at $\mathrm{pH}$ 7.6.

Common features of the cyclic voltammograms obtained at both type of electrodes are: (i) the ratio of 


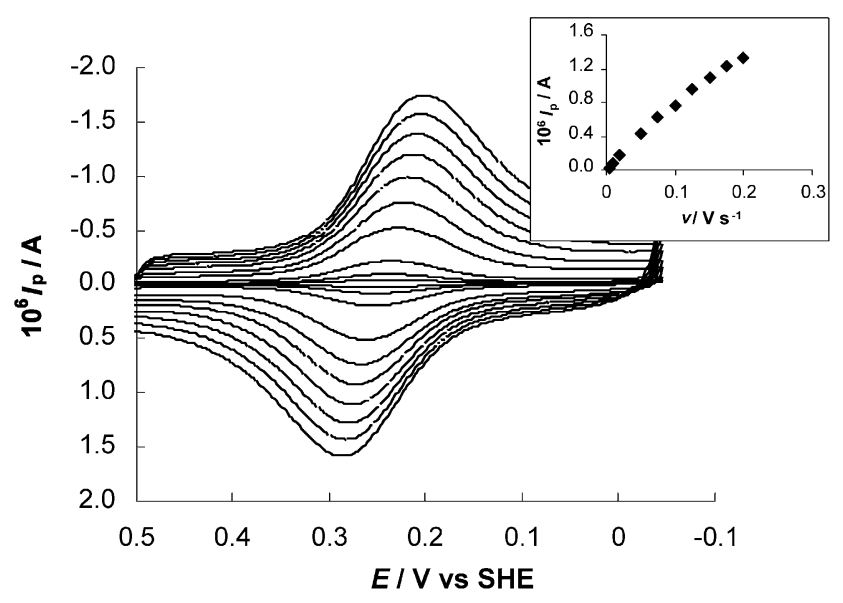

Fig. 1. Cyclic voltammograms of $100 \mu \mathrm{M}$ cytochrome $c_{552}$ at the membrane PGE in $10 \mathrm{mM}$ Tris chloride buffer solution ( $\mathrm{pH}$ 7.6). Scan rate $\left(\mathrm{mV} \mathrm{s}^{-1}\right): 5,10,20,50,75,100,125,150,175,200$. Insert: variation of $\mathrm{CV}$ cathodic peak current with the scan rate.

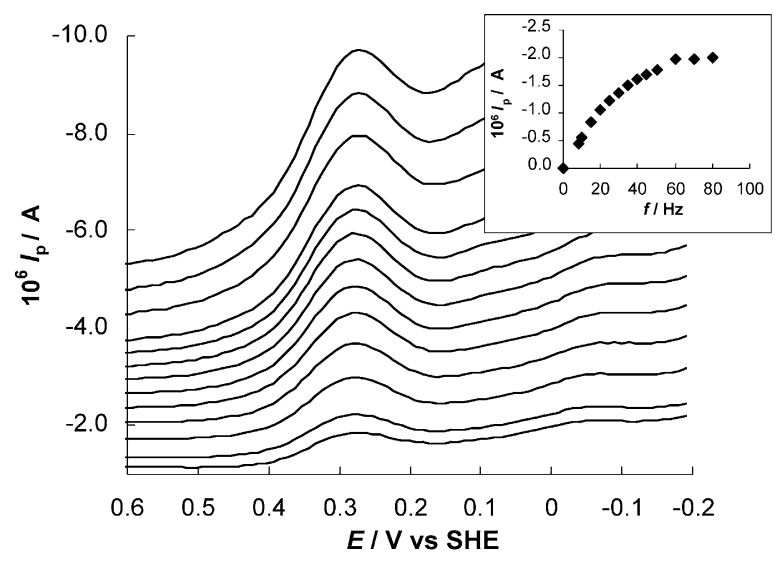

Fig. 2. Square wave voltammograms of $100 \mu \mathrm{M}$ cytochrome $c_{552}$ at the membrane GCE in $10 \mathrm{mM}$ Tris chloride buffer solution (pH 7.6). Frequency (Hz): 8, 10, 15, 20, 25, 30, 35, 40, 45, 60, 70, 80, 90, 100. Insert: variation of SW peak current with the frequency.

the cathodic, $I_{\mathrm{pc}}$, and anodic, $I_{\mathrm{pa}}$, peak currents is close to one for all scan rates; (ii) $I_{\mathrm{pc}}$ and $I_{\mathrm{pa}}$ increase linearly with increasing scan rate, at least up to a certain value (typically $100 \mathrm{mV} \mathrm{s}^{-1}$; see insert of Fig. 1) and (iii) the difference, $\Delta E_{\mathrm{p}}$, between the cathodic, $E_{\mathrm{pc}}$, and the anodic, $E_{\mathrm{pa}}$, peak potentials, $\Delta E_{\mathrm{p}}=E_{\mathrm{pa}}-E_{\mathrm{pc}}$, increases with increasing scan rate, though it tends to zero when the scan rate decreases. Over the range of scan rates analyzed, $\left(\Delta E_{\mathrm{pc}}+E_{\mathrm{pa}}\right) / 2$ values are constant within the experimental errors $( \pm 5 \mathrm{mV})$.

In SW voltammetry the peak current, $I_{\mathrm{p}}$, initially increases with the increase of frequency but then tends to a constant value (see insert of Fig. 2). As for the peak potentials, $E_{\mathrm{p}}$, as well as the peak width at half height, $W_{1 / 2}$, they remained constant in the frequency range studied.

Similar behavior was found in cyclic and SW voltammetry for the reduction of cytochrome $c_{553}$ at the same
MEs and under the same experimental conditions (not shown).

DP was also used in some experiments. It is observed that for different pulse durations, $t_{\mathrm{p}}$, the peak potential, $E_{\mathrm{p}}$, and its width at half height, $W_{1 / 2}$, remain constant and that the peak current, $I_{\mathrm{p}}$, increases with $1 / t_{\mathrm{p}}$.

In Table 1, peak potentials, half height width of SW and DP voltammograms and $\left(E_{\mathrm{pc}}+E_{\mathrm{pa}}\right) / 2$ values extracted from $\mathrm{CV}$ are summarized.

The electrochemistry of both cytochromes was then analyzed at the membrane gold electrode (MAuE) not previously modified. Aliquots of both cytochromes in the buffer solution were entrapped between the membrane and the AuE surface and the MAuE immersed just in the Tris buffer. In Fig. 3, typical cyclic (A) and square wave (B) voltammograms obtained at the MAuE are shown for $110 \mu \mathrm{M}$ cytochrome $c_{533}$ solution. Stable and reproducible voltammograms were always obtained for both cytochromes (data for $c_{552}$ not shown) either in $\mathrm{CV}$, SW or DP voltammetry. Analysis of the voltammograms in terms of the current and peak dependence on scan rate, frequency and pulse duration (depending on the technique) revealed that the reduction of cytochromes $c_{522}$ and $c_{533}$ at the MAuE closely follows the behavior previously described at the MGCE and PGEs. In Table $1,\left(E_{\mathrm{pc}}+E_{\mathrm{pa}}\right) / 2$ values from $\mathrm{CV}$, as well as $E_{\mathrm{p}}$ and $W_{1 / 2}$ values from SW and DP voltammograms are summarized.

Further analysis of the CV data indicates a quasireversible redox reaction following thin layer theory [31]. Namely, the peak current increases linearly with $v$, in contrast to the diffusion controlled behavior (linear $I_{\mathrm{p}}$ vs. $v^{1 / 2}$ plot), and the cathodic and anodic peak separation increases with increasing scan rates. For all the scan rates, $\left(E_{\mathrm{pa}}+E_{\mathrm{pc}}\right) / 2$ values are constant within the experimental errors and so a measure of $E^{\circ \prime}$ can be evaluated as the mean of $E_{\mathrm{pc}}$ and $E_{\mathrm{pa}}$.

An estimation of the volume $V\left(\mathrm{~cm}^{3}\right)$ of effectively electrolyzed solution at the ME can be calculated using the concepts of thin layer voltammetry. Considering that the redox reactions are in the boundary of reversible behavior, $V$ can be computed through the dependence of either the cathodic or anodic $\mathrm{CV}$ peak currents, $I_{\mathrm{p}}$ (A), with the scan rate $v\left(\mathrm{~V} \mathrm{~s}^{-1}\right)$ using the relationship [31]:

$I_{\mathrm{p}}=\left(9.39 \times 10^{5}\right) n^{2} V c v$

In Eq. (1), $c\left(\mathrm{~mol} \mathrm{~cm}^{-3}\right)$ is the concentration of the entrapped protein solution, $n$ the number of electrons exchanged in the redox reaction and the other symbols have the meaning previously defined. Taking into account the areas of the electrodes, $A\left(\mathrm{~cm}^{2}\right)$, a typical layer thickness, $l(\mathrm{~cm})$, can be computed for each experiment. The values thus obtained are shown in Tables 2 and 3. 
Table 1

Electrochemical behavior of the proteins at the membrane electrodes

\begin{tabular}{|c|c|c|c|c|c|c|c|c|c|}
\hline \multirow{2}{*}{$\begin{array}{l}\text { Membrane electrode } \\
\text { Protein }\end{array}$} & \multirow[t]{2}{*}{ Parameter } & \multicolumn{3}{|c|}{ GCarbon } & \multicolumn{3}{|c|}{ Gold } & \multicolumn{2}{|c|}{ PGraphite } \\
\hline & & $\mathrm{CV}$ & SW & DP & $\mathrm{CV}$ & SW & DP & $\mathrm{CV}$ & SW \\
\hline P. nautica & $\left(E_{\mathrm{pc}}+E_{\mathrm{pa}}\right) / 2$ or $E_{\mathrm{p}} / \mathrm{mV}$ & 227 & 228 & - & 222 & 223 & 214 & 244 & 247 \\
\hline$c_{552}$ & $W_{1 / 2} / \mathrm{mV}$ & - & 126 & - & - & 126 & 112 & - & 119 \\
\hline D. vulgaris & $\left(E_{\mathrm{pc}}+E_{\mathrm{pa}}\right) / 2$ or $E_{\mathrm{p}} / \mathrm{mV}$ & 18 & 25 & 23 & 13 & 13 & 7 & 16 & 23 \\
\hline$c_{553}$ & $W_{1 / 2} / \mathrm{mV}$ & - & 131 & 105 & - & 117 & 94 & - & 131 \\
\hline D. vulgaris ${ }^{\mathrm{a}}$ & $\left(E_{\mathrm{pc}}+E_{\mathrm{pa}}\right) / 2$ or $E_{\mathrm{p}} / \mathrm{mV}$ & - & - & - & - & - & - & 30 & 33 \\
\hline Rubredoxin & $W_{1 / 2} / \mathrm{mV}$ & - & - & - & - & - & - & - & 126 \\
\hline
\end{tabular}

Peak potentials and peak width at half height are within the experimental error $\pm 5 \mathrm{mV}$. Medium: Tris buffer 0.01 $\mathrm{M} \mathrm{pH} 7.6$.

a Tris buffer $0.01 \mathrm{M} \mathrm{pH} \mathrm{7.6,} \mathrm{KCl} 0.1 \mathrm{M}$ and poly-L-lysine.
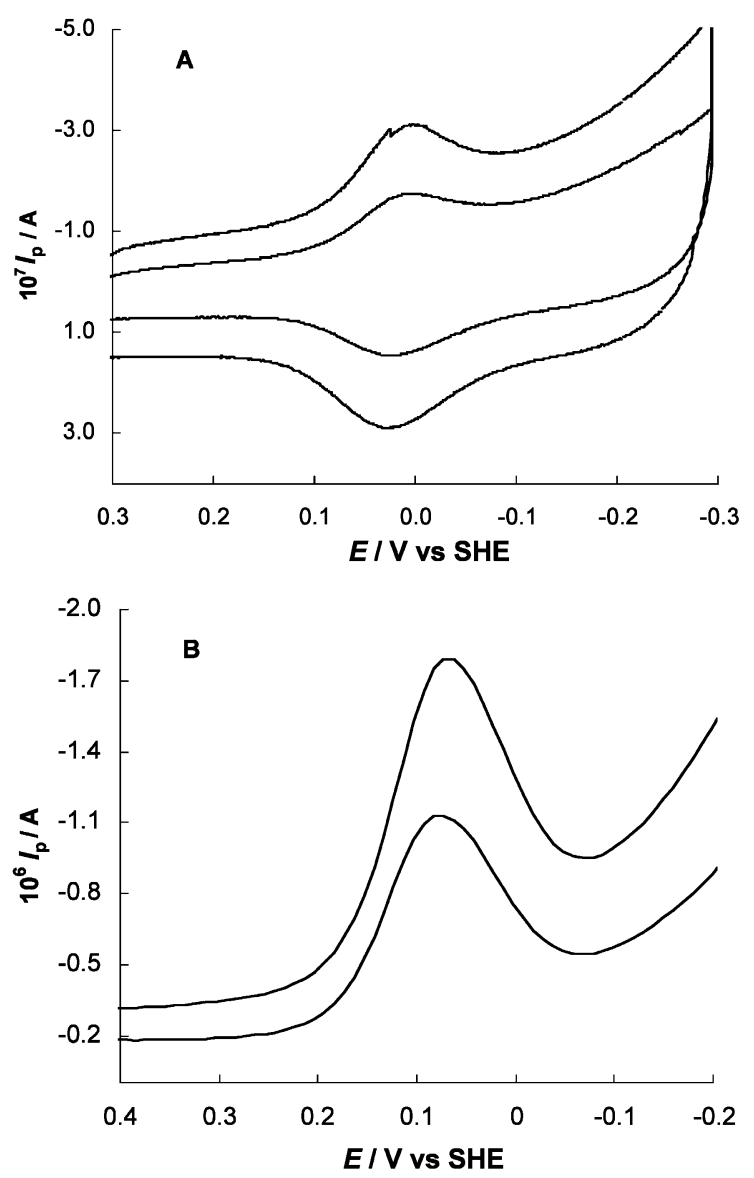

Fig. 3. Cyclic voltammograms (A) and square wave voltammograms (B) of $110 \mu \mathrm{M}$ cytochrome $c_{553}$ at the MAuE in $10 \mathrm{mM}$ Tris chloride buffer (pH 7.6). $\mathrm{CV}$, scan rate $\left(\mathrm{mV} \mathrm{s}^{-1}\right)$ : 50, 100; $\mathrm{SW}$, frequency $(\mathrm{Hz})$ : 8,15 .

Using Laviron's treatment for thin layer voltammetry [32], the heterogeneous charge transfer rate constant, $k_{\mathrm{s}}$ $\left(\mathrm{cm} \mathrm{s}^{-1}\right)$ can be calculated from the variation of the peak potential separation with the scan rate fitted to a working function of $\Delta E_{\mathrm{p}}$ versus the kinetic parameter $m$ :

$m=k_{\mathrm{s}} A /[V(n F / R T) v]$
The values computed are shown in Tables 2 and 3. In the same tables are also presented, for comparison, $k_{\mathrm{s}}$ as well as $E^{\circ \prime}$ values obtained with non-MEs. All these values will be discussed later.

A comment should be made, however, about $l$. Although $l$ values depend on experimental parameters, especially on the preparation of the ME, fairly reproducible results were obtained as can be seen in Tables 2 and 3. Thin layer behavior will be observed as long as the cell thickness $l$ is smaller than the diffusion layer thickness for a given experimental time scale, $t$, that is:

$l<(2 D t)^{1 / 2}$

where $D$ is the diffusion coefficient of the species. Using $D=1.5 \times 10^{-6} \mathrm{~cm}^{2} \mathrm{~s}^{-1}$ as a mean value for the diffusion coefficient of the monohemic cytochromes [21] and for the highest $l$ found experimentally $(l=$ $10^{-3} \mathrm{~cm}$, Table 3) this corresponds to $t=0.3 \mathrm{~s}$. So, in $\mathrm{CV}$ mass transfer within the cell can be neglected and thin layer behavior observed for scan rates up to about $R T / F t=0.09 \mathrm{~V} \mathrm{~s}^{-1}$. In SW and DP voltammetry experiments, the diffusion layer thickness is always smaller than $l$, even for the longest time scales used, i.e. $t_{\mathrm{p}}=0.1 \mathrm{~s}(f=5 \mathrm{~Hz})$ and $t_{\mathrm{p}}=0.075 \mathrm{~s}$, respectively. Analysis of SW and DP voltammograms should be made in terms of diffusion-controlled processes, which shows that the reductions of cytochrome $c_{522}$ and $c_{533}$ at the ME are within the boundaries of reversibility. Indeed, values of peak width at half heights of both SW and DP voltammograms do not depart significantly from those expected for reversible processes. For $n=1$, the theoretical values are $W_{1 / 2}=91 \mathrm{mV}$ and $W_{1 / 2}=123$ $\mathrm{mV}$ for DP and SW voltammetry, respectively, [31,33]. Peak potentials remained constant within the experimental error $( \pm 5 \mathrm{mV})$ over the frequency and $t_{\mathrm{p}}$ ranges analyzed. So, $E_{\mathrm{p}}$ values from SW voltammograms can be used as a direct measure of formal potentials $E^{\circ \prime}$. As far as DP voltammograms are concerned one has $E^{\circ \prime}=$ $E_{\mathrm{p}}+\Delta E / 2$, where $\Delta E$ is the pulse amplitude. The values obtained are summarized in Tables 2 and 3. 
Table 2

Results for cytochrome $c_{552}$ from P. nautica

\begin{tabular}{|c|c|c|c|c|c|}
\hline Electrode $(d / \mathrm{mm})$ & $E^{\circ \prime} \pm 5 / \mathrm{mV}$ vs. SHE & $10^{3} k_{\mathrm{s}} / \mathrm{cm} \mathrm{s}^{-1}$ & $10^{5} / \mathrm{V} \mathrm{cm}^{-3}$ & $10^{4} l / \mathrm{cm}$ & Reference \\
\hline $\mathrm{GC}, d=2.8^{\mathrm{a}}$ & 215 & $5.0 \pm 0.1$ & - & - & {$[21]$} \\
\hline $\mathrm{GC}, d=0.006^{\mathrm{a}}$ & 228 & $2.0 \pm 0.1$ & - & - & {$[21]$} \\
\hline $\mathrm{Au}, d=1.6^{\mathrm{a}}$ & - & $0.8 \pm 0.1$ & - & - & {$[21]$} \\
\hline $\mathrm{Au}, d=0.022^{\mathrm{a}}$ & 233 & $0.4 \pm 0.1$ & - & - & {$[21]$} \\
\hline $\mathrm{MPG}, d=4^{\mathrm{b}}$ & $244,247(\mathrm{SW})$ & $1.4 \pm 0.3$ & $7 \pm 1$ & $5.8 \pm 0.5$ & This work \\
\hline $\mathrm{MGC}, d=3^{\mathrm{b}}$ & $227,228(\mathrm{SW})$ & $1.2 \pm 0.2$ & $1.6 \pm 0.1$ & $2.2 \pm 0.2$ & This work \\
\hline $\mathrm{MAu}, d=1.5^{\mathrm{b}}$ & 222,223 (SW), 214 (DP) & $1.0 \pm 0.2$ & $0.8 \pm 0.1$ & $4.5 \pm 0.5$ & This work \\
\hline
\end{tabular}

All values shown were computed from CV data, unless otherwise specified: SW square wave and DP differential pulse.

a Medium: phosphate buffer and 4,4'-dipyridyl.

b Medium: Tris buffer and membrane $\mathrm{M}^{-}$Spectra/Por.

One of the advantages of the ME approach is the capacity to investigate rapidly the influence of various experimental parameters on the redox behavior, like the $\mathrm{pH}$ and the composition of the supporting electrolyte. This can be done without using large amounts of protein samples since the ME, once mounted in the cell, need not to be removed and serves for all the experiments.

The $\mathrm{pH}$ dependence of the formal potential $E^{\circ \prime}$ and peak currents of cytochrome $c_{553}$ was investigated using SW voltammetry at the MEs. The results obtained for the variation of $E^{\circ \prime}$ at the MPGE are shown in Fig. 4, where SW peak potentials were used as a measure of $E^{\circ \prime}$, as discussed before. In the same figure is also shown the $\mathrm{pH}$ dependence of the formal potential as determined by $\mathrm{CV}$ measurements at a gold electrode in the presence of 4,4'-dithiodipyridine as reported before [21].

The square wave peak current dependence on $\mathrm{pH}$ at the MPGE can be seen in the insert of Fig. 4. The variation observed for cytochrome $c_{553}$ follows previous results obtained by $\mathrm{CV}$ at a 4,4'-bipyridine modified gold electrode. In particular, the marked decrease above $\mathrm{pH} 10-11$ due to a modification of the cytochrome $c_{553}$ structure is observed, as discussed in Ref. [11].

From this set of results and as previously reported for horse heart cytochrome $c$ at a MPGE [25], MEs furnished the same formal potential-current-pH profiles as those observed at conventional electrodes and in a more efficient way.

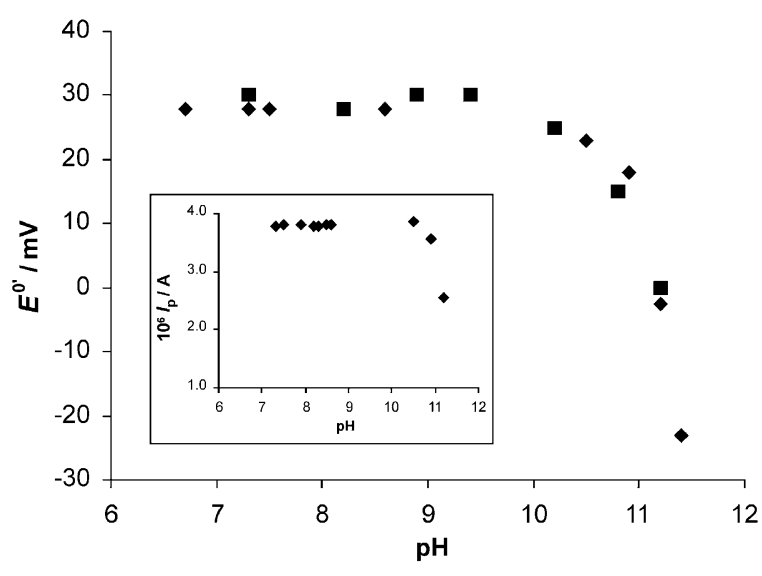

Fig. 4. $\mathrm{pH}$ dependence of the formal potential $E^{\circ}$ of cytochrome $c_{553}$ determined by SW voltammetry at the membrane GCE $(\diamond)$ and $\mathrm{CV}$ at

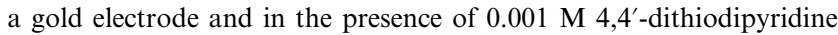
(ם). Insert: $\mathrm{pH}$ dependence of the peak current for cytochrome $c_{553}$ determined by SW voltammetry at the membrane GCE.

The effect of varying the ionic strength of the supporting electrolyte was analyzed for both cytochromes at the MAu and MPG electrodes following the variation of the SW peak currents upon additions of $\mathrm{NaNO}_{2}$ and $\mathrm{NaCl}$. The results obtained at the MAu and MPG electrodes can be seen in Fig. 5(A) and (B), respectively. While the peak current height at the MAuE decreases with the increase of both $\mathrm{NaCl}$ and $\mathrm{NaNO}_{2}$

Table 3

Results for cytochrome $c_{553}$ from $D$. vulgaris

\begin{tabular}{|c|c|c|c|c|c|}
\hline Electrode $(d / \mathrm{mm})$ & $E^{\circ \prime} \pm 5 / \mathrm{mV}$ vs. SHE & $10^{3} k_{\mathrm{s}} / \mathrm{cm} \mathrm{s}^{-1}$ & $10^{5} / V \mathrm{~cm}^{-3}$ & $10^{4} / / \mathrm{cm}$ & Reference \\
\hline $\mathrm{Au}, d=0.5^{\mathrm{a}}$ & 20 & $\approx 1$ & - & - & [11] \\
\hline $\mathrm{GC}, d=0.006^{\mathrm{b}}$ & 4 & - & - & - & [21] \\
\hline $\mathrm{Au}, d=0.022^{\mathrm{b}}$ & $11,14(\mathrm{SW}), 8$ (DP) & - & - & - & [21] \\
\hline $\mathrm{MGC}, d=3^{\mathrm{c}}$ & $18,25(\mathrm{SW}), 23(\mathrm{DP})$ & $5 \pm 1$ & $5 \pm 1$ & $7 \pm 1$ & This work \\
\hline $\mathrm{MAu}, \mathrm{d}=1.5^{\mathrm{c}}$ & 13, $14(\mathrm{SW}), 7(\mathrm{DP})$ & $7 \pm 1$ & $1 . \overline{8} \pm 0.2$ & $10 \pm 1$ & This work \\
\hline
\end{tabular}

\footnotetext{
All values shown were computed from $\mathrm{CV}$ data, unless otherwise specified: SW, square wave and DP, differential pulse.

${ }^{a}$ Medium: phosphate buffer and 4,4'-dipyridine.

b Medium: phosphate buffer and 4,4'-dipyridyl.

${ }^{c}$ Medium: Tris buffer and membrane $\mathrm{M}^{-}$Spectra/Por.
} 

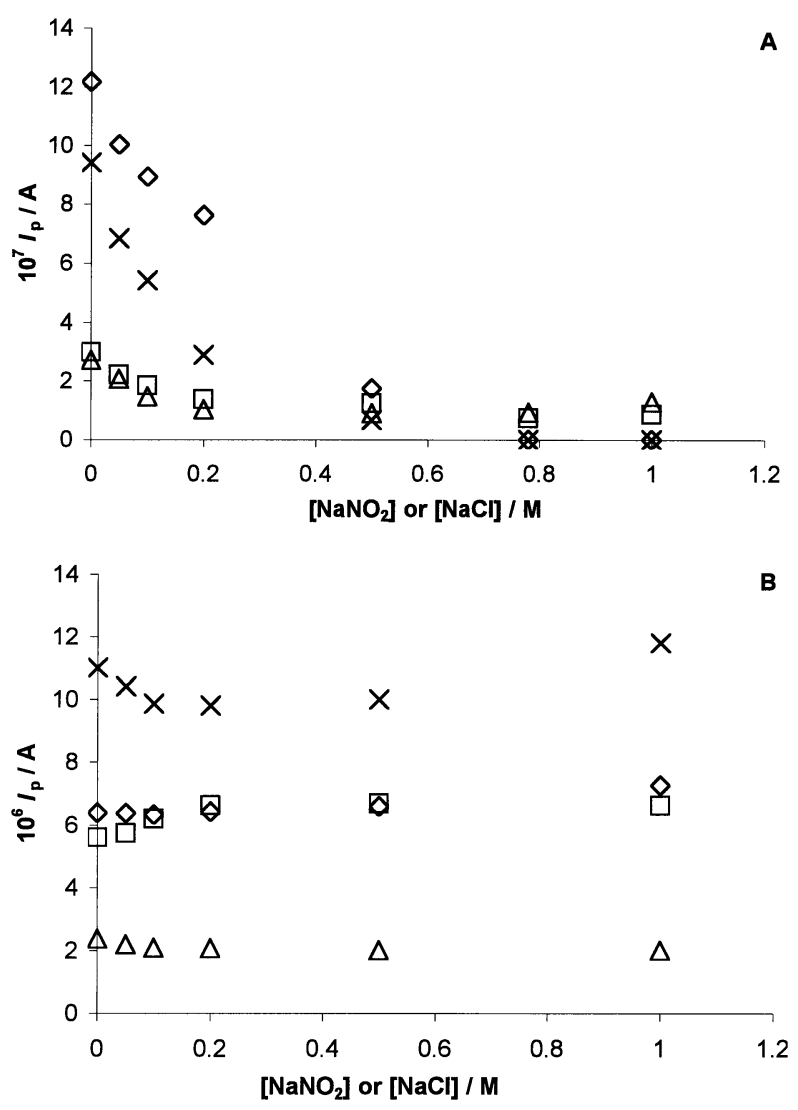

Fig. 5. Ionic strength dependence of SW peak current in $10 \mathrm{mM}$ Tris chloride buffer ( $\mathrm{pH}$ 7.6): (A) at the MAuE for $110 \mu \mathrm{M}$ cytochrome $c_{553}$ in the presence of $(\diamond) \mathrm{NaNO}_{2}$ and $(\times) \mathrm{NaCl}$ and for $100 \mu \mathrm{M}$ cytochrome $c_{552}$ in the presence of $(\square) \mathrm{NaNO}_{2}$ and $(\Delta) \mathrm{NaCl}$; (B) at the membrane PGE for $110 \mu \mathrm{M}$ cytochrome $c_{553}$ in the presence of $(\diamond)$ $\mathrm{NaNO}_{2}$ and $(\times) \mathrm{NaCl}$ and for $100 \mu \mathrm{M}$ cytochrome $c_{552}$ in the presence of $(\square) \mathrm{NaNO}_{2}$ and $(\Delta) \mathrm{NaCl}$.

concentrations, constant SW peak heights (within 5\%) were obtained at the MPGE. As to the SW peak potentials, in most situations there is some shift towards less positive values upon increase of the salt concentration, as can be appreciated in Fig. 6 .

\subsection{Electrochemistry of rubredoxin at MEs}

Typical cyclic voltammograms obtained at the MPGE are shown in Fig. 7 for $71 \mu \mathrm{M}$ rubredoxin entrapped between the membrane and the PG electrode in the absence (A) and in the presence (B) of poly-L-lysine (PLL). The MPGE was immersed in $0.1 \mathrm{M} \mathrm{KCl}$ and Tris buffer $\mathrm{pH}$ 7.6. As can be seen from Fig. 7, a voltammetric response is obtained only in the presence of PLL. This confirms previous work where it was shown that the presence of positively charged species is a mandatory condition for the occurrence of reduction of negatively charged proteins at an electrode that bears a net negative charge $[15,16]$.

Analysis of the cyclic voltammograms for scan rates in the range $15-200 \mathrm{mV} \mathrm{s}^{-1}$, showed that the depen-
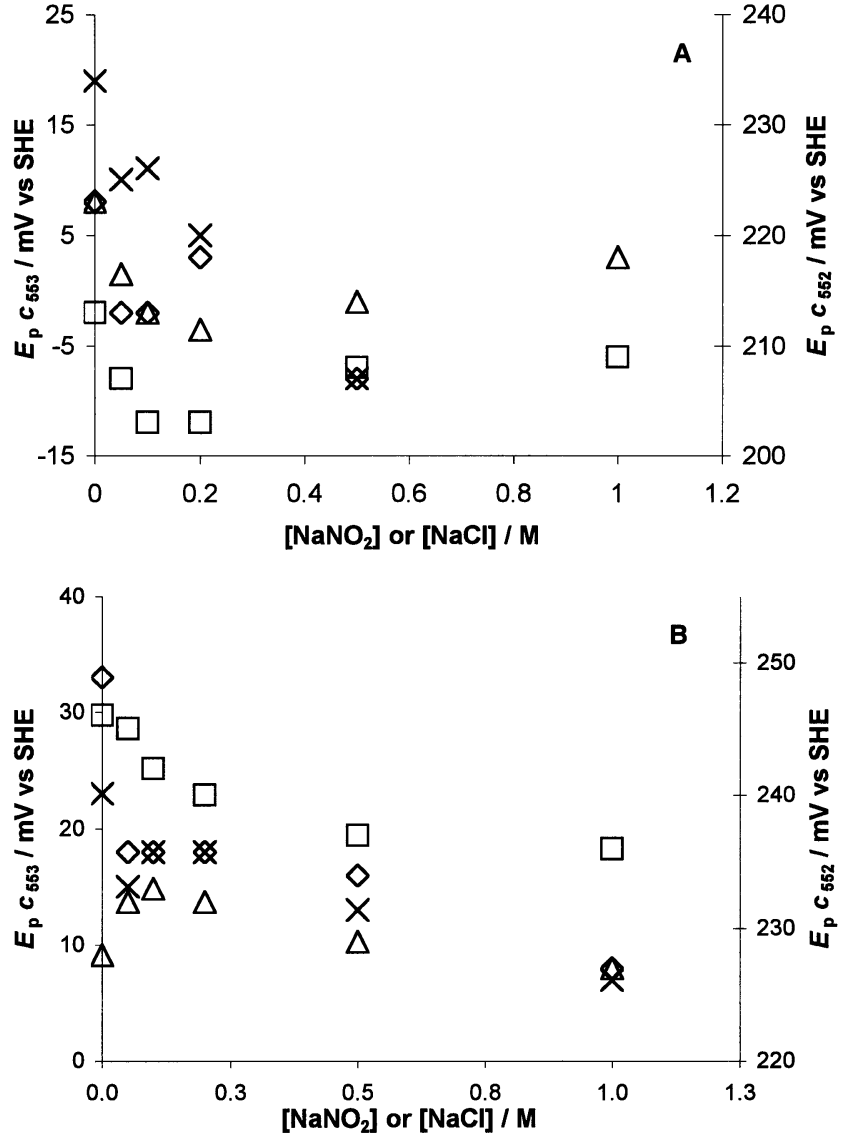

Fig. 6. Ionic strength dependence of SW peak potential in $10 \mathrm{mM}$ Tris chloride buffer ( $\mathrm{pH}$ 7.6): (A) at the MAuE for $110 \mu \mathrm{M}$ cytochrome $c_{553}$ in the presence of $(\diamond) \mathrm{NaNO}_{2}$ and $(\times) \mathrm{NaCl}$ and for $100 \mu \mathrm{M}$ cytochrome $c_{552}$ in the presence of $(\square) \mathrm{NaNO}_{2}$ and $(\triangle) \mathrm{NaCl}$; (B) at the membrane PGE for $110 \mu \mathrm{M}$ cytochrome $c_{553}$ in the presence of $(\diamond)$ $\mathrm{NaNO}_{2}$ and $(\times) \mathrm{NaCl}$ and for $100 \mu \mathrm{M}$ cytochrome $c_{552}$ in the presence of $(\square) \mathrm{NaNO}_{2}$ and $(\triangle) \mathrm{NaCl}$

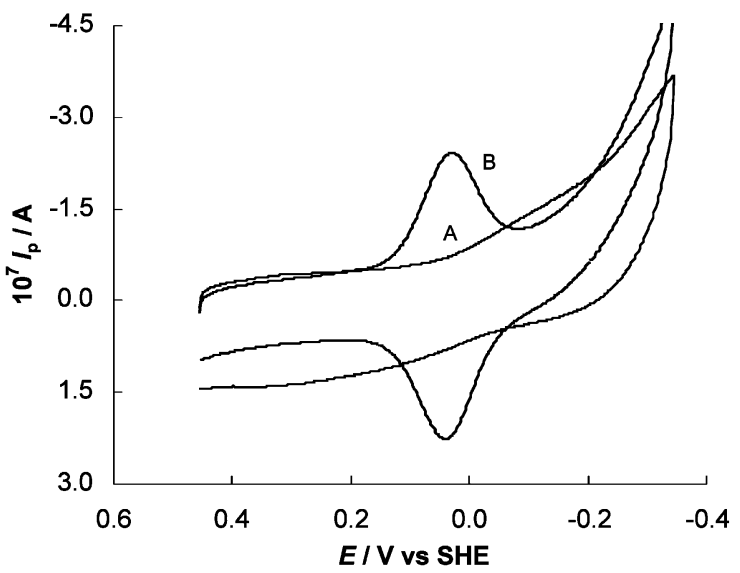

Fig. 7. Cyclic voltammograms of $71 \mu \mathrm{M}$ rubredoxin at the membrane PGE in $0.1 \mathrm{M} \mathrm{KCL}$ and $10 \mathrm{mM}$ Tris chloride buffer solution (pH 7.6) in the absence (A) and presence (B) of $130 \mu \mathrm{g} \mathrm{cm}^{-3}$ of poly-L-lysine. Scan rate $\left(\mathrm{mV} \mathrm{s}^{-1}\right): 20$

dence of $I_{\mathrm{pc}}$ and $I_{\mathrm{pa}}$ on $v$ is linear for scan rates $<100$ $\mathrm{mV} \mathrm{s}^{-1}$, in accord with thin layer behavior. Moreover, 
the difference between the anodic and cathodic peak potentials increases with an increase in the scan rate, from $10 \mathrm{mV}$ at $5 \mathrm{mV} \mathrm{s}^{-1}$ to $57 \mathrm{mV}$ at $200 \mathrm{mV} \mathrm{s}^{-1}$, thus indicating a quasi-reversible process. Constant $\left(E_{\mathrm{pa}}+\right.$ $\left.E_{\mathrm{pc}}\right) / 2$ values within the experimental errors were obtained for all scan rates.

Well-defined and reproducible SW voltammograms were also obtained at the MPGE in the presence of PLL, with $W_{1 / 2}=126 \mathrm{mV}$ and $E_{\mathrm{p}}=33 \mathrm{mV}$.

Further analysis of the data, as previously described for the monohemic cytochromes, leads to the values of $E^{\circ \prime}, k, V$ and $l$ collected in Table 4 . In this table $E^{\circ \prime}$ and $k_{\mathrm{s}}$ values reported in the literature are also presented for comparison.

\section{General discussion}

From the data presented in Tables 2-4 two major facts are apparent. Clearly, good agreement is achieved for the formal potentials as well as the heterogeneous charge transfer rate constant values obtained at the different MEs. Moreover, and in spite of all the factors that can influence a successful communication between a redox protein and a suitable electrochemical interface, it is worth noticing how the electrochemical data at the $\mathrm{ME}$ for this set of proteins matches previous results. Similar values were obtained for $E^{\circ \prime}$ and $\mathrm{k}_{\mathrm{s}}$ using either different types of MEs (MPG, MGC, MAu) or nonMEs of gold, carbon and ruthenium, with dimensions ranging from the $\mu \mathrm{m}$ to the $\mathrm{mm}$ size, and in the absence or in the presence of different facilitators or surface modifiers.

However, the results now presented at the MAuE, namely for cytochrome $c_{553}$, differ from previous reports in the sense that, in this work, a direct electrochemical response of both $D$. vulgaris cytochrome $c_{533}$ and $P$. nautica cytochrome $c_{522}$ was observed when a thin layer of protein solution was entrapped between the negatively charged membrane and the gold electrode surface in the absence of any sort of facilitator.

It has been claimed that in order to achieve a fast electron transfer between a redox protein and a metal electrode, the surface needs to be functionalized in such a way as to allow a protein-electrode surface recognition process to take place. The results now presented, as well as those reported for $T$. ferrooxidans cytochrome $c_{552}$ and horse heart cytochrome $c$ reductions at similar PM gold electrodes [23,25], suggest a mechanism involving mainly favorable electrostatic interactions between the charged redox proteins and the opposite charges of the membrane. Reasonably fast, reproducible and persistent responses were obtained in a hydrophobic surface not previously modified or in the presence of some facilitator. Even undesirable phenomena like blocking of the electrode surface by impurities or the proteins themselves seem to be overridden by the use of a charged membrane close to the electrode surface. Direct electrochemical communication between the redox proteins and the electrode certainly results from a proper orientation of the cytochromes governed by the electric environment built up by the charge membrane.

Clear evidence that electrostatic interactions are implied in the redox reactions of the above proteins at the MAuE is shown up by how the ionic strength of the medium affects the electrochemical response. For both cytochromes, and as shown in Fig. 5, there is a decrease of SW peak currents with the increase of the salt concentration, independent of the nature of the anion of the electrolyte (which eventually could have a different affinity to the positively charged sites of both cytochromes). Increasing the ionic strength causes a diminishing of the overall charge of both the membrane and the cytochromes thus decreasing the favorable electrostatic interactions. Since the overall charge as well as the dipolar moment for cytochrome $c_{553}$ is higher than for cytochrome $c_{552}(+9$ and $322 \mathrm{D}$, and +4 and $215 \mathrm{D}$, respectively) the effect is much more pronounced for the former.

The decrease of the SW peak currents is accompanied by a negative shift tendency of the SW peak potential, as can be seen in Fig. 6, showing that the redox reaction becomes more difficult. This variation is again more pronounced for cytochrome $c_{553}$.

The results now presented at the membrane carbon electrodes show that the presence of a negatively

Table 4

Results for rubredoxin from D. vulgaris

\begin{tabular}{|c|c|c|c|c|c|}
\hline Electrode $(d / \mathrm{mm})$ & $E^{\circ \prime} / \mathrm{mV} \pm 5$ vs. SHE & $10^{3} k_{\mathrm{s}} / \mathrm{cm} \mathrm{s}^{-1}$ & $10^{4} / V \mathrm{~cm}^{-3}$ & $10^{4} l / \mathrm{cm}$ & Reference \\
\hline $\mathrm{Au}, d=1.6^{\mathrm{a}}$ & 5 & $0.7 \pm 0.2$ & - & - & {$[22]$} \\
\hline $\mathrm{Au}, d=0.025^{\mathrm{a}}$ & 8 & $0.7 \pm 0.3$ & - & - & {$[22]$} \\
\hline $\mathrm{GC}, d=3^{\mathrm{a}}$ & 16 & $1.0 \pm 0.5$ & - & - & [22] \\
\hline $\mathrm{MPG}, d=4^{\mathrm{b}}$ & $30,33(\mathrm{SW})$ & $3.8 \pm 0.3$ & $(1.2 \pm 0.1)$ & $(9.5 \pm 0.8)$ & This work \\
\hline
\end{tabular}

All values shown were computed from $\mathrm{CV}$ data, unless otherwise specified: SW, square wave and DP, differential pulse.

a Medium: $0.1 \mathrm{M} \mathrm{KCl}$, Tris buffer, neomycine and $\mathrm{Mg}^{2+}$.

b Medium: $0.1 \mathrm{M} \mathrm{KCl}$, Tris buffer, poly-L-lysine and membrane $\mathrm{M}^{-}$Spectra/Por. 
charged membrane in close vicinity to the electrode surfaces is effective in entrapping the protein solutions while not disturbing the environment of the imprisoned electroactive species. Indeed, the behavior reported at the MPGE and MGCE for both cytochromes follows previous work more closely, namely a quasi-reversible response was observed for the reduction of cytochrome $c_{553}$ at a bare PGE in the absence of any activating agent [34] and the same has been reported for horse heart cytochrome $c$. The isoelectric points of both cytochromes $c_{522}$ and $c_{533}$ are not as high as that of cytochrome $c(\mathrm{p} I=10.5)$ and so the overall positive charge is not so determining, but still important interactions must be operative with the carbon surfaces negatively charged through deprotonation of acidic $\mathrm{C}-$ $\mathrm{O}$ functionalities. However, electrostatic effects do not seem to be the major factor determining the electron transfer process since the redox behavior of both cytochromes at the membrane PGE was shown to be much more insensitive to changes in ionic strength. There is some effect on the SW peak potentials, again clearer for cytochrome $c_{553}$ (Fig. 6) but, in contrast to what was observed at the gold ME, constant SW peak currents were obtained within $5 \%$ for $\mathrm{NaNO}_{2}$ or $\mathrm{NaCl}$ concentrations up to $1 \mathrm{M}$, as can be seen in Fig. 5. This does not rule out that discrete charge interactions are absent but other complementary interactions must be more important. Hydrogen-bonding interactions between the electrode surface $\mathrm{C}-\mathrm{O}$ functionalities and most probably lysine groups on cytochromes must be the major factor controlling the efficiency of the electron transfer. Both model building and experimental approaches $[35,36]$ show that interactions between lysine residues and carboxylates on a reaction partner are important features of the physiological situation. These features are mimicked by the interactions of the $\mathrm{C}-\mathrm{O}$ functionalities with the protein and a proper orientation towards the electrode surface is then achieved and the electron transfer occurs.

As to the results obtained at the MPGE for rubredoxin, which presents an overall negative charge of -13 at $\mathrm{pH}$ close to 7, the absence of a response in the absence of a positively charge species can be clearly identified with electrostatic barrier at the negatively charged electrode surface: only by inclusion in the entrapped protein solution of poly-L-lysine does the reduction reaction take place. These results follow from previous results on the electrochemistry of rubredoxin at carbon electrodes [12,23] as well as from what has been observed for $[2 \mathrm{Fe}-2 \mathrm{~S}]$ spinach ferredoxin entrapped between a negative membrane and a graphite surface [24]. In any case, once a suitable positive species is included in the protein solution, reduction occurs and the negative charge of the membrane seems not to affect the redox reaction of the negatively charged protein. So, ME remained very attractive for the study of these redox systems since aspects like the very tiny amounts of protein needed and how easily several experimental variables can be changed using the same unit are still valid.

\section{Conclusions}

Many effects can modulate the electrochemical behavior of redox proteins at metal and non-metal electrodes. The good agreement obtained in all situations for the electrochemical parameters, using different strategies, helped to gain a further insight into the nature and relative importance of all the factors that can affect charge transfer in complex redox systems like proteins. Different electrodes and experimental conditions were used: metals and non-metals, micro to millimeter size range, absence and presence of facilitators. However, in all cases a favorable domain for electron transfer to occur was certainly achieved which accounts for the similarity of the values encountered for the formal potentials as well as for the heterogeneous charge transfer rate constants. Discrepancies in the literature between, for instance, $k_{\mathrm{s}}$ values have been attributed to how critically dependent they are on the nature of the electrode $\mid$ solution interface. The results discussed show that the electrochemical response of this set of proteins is not very dependent on factors such as the nature of the electrode material, as long as a suitable domain for electron transfer is built up at the electrode solution interface by different types of complementary interactions. The results presented in this work of the direct electrochemistry of the cytochromes at the MAuE, together with previous data, show that electrostatic interactions can indeed be very important in determining electron transfer reactions of these biological molecules.

Although most redox reactions in the biological environment are highly specific, binding interactions are to be expected where there are complementary domains to engage multi-site interactions. This can be mimicked by different types of electrochemical interfaces that are then capable of interacting reversibly and specifically with the protein surface, thus creating a favorable domain for electron transfer to occur.

\section{Acknowledgements}

This work is within the research project POCTI/QUI/ 42277/2001. P.M.P. thanks for financial support from FCT. 


\section{References}

[1] P. Yeh, T. Kuwana, Chem. Lett. (1977) 1145.

[2] M.J. Eddowes, H.A.O. Hill, J. Chem. Soc. Chem. Commun. (1977) 771.

[3] F.A. Armstrong, H.A.O. Hill, N.J. Walton, Acc. Chem. Res. 21 (1988) 407.

[4] H.A.O. Hill, N.I. Hunt, Methods in Enzymology, vol. 227 (Ch. 19), Academic Press, New York, 1993.

[5] P. Bianco, J. Haladjian, Biochimie 76 (1994) 605.

[6] F.A. Armstrong, G.S. Wilson, Electrochim. Acta 45 (2000) 2623.

[7] F.N. Buchi, A.M. Bond, J. Electroanal. Chem. 314 (1991) 191

[8] H.A.O. Hill, N.I. Hunte, A.M. Bond, J. Electroanal. Chem. 436 (1997) 17.

[9] P.M. Allen, H.A.O. Hill, N.J. Walton, J. Electroanal. Chem. 178 (1984) 69.

[10] H.A.O. Hill, D.J. Page, N.J. Walton, D. Whitford, J. Electroanal. Chem. 187 (1985) 315.

[11] P. Bianco, J. Haladjian, R. Pilard, J. Electroanal. Chem. 136 (1982) 291

[12] F.A. Armstrong, P.A. Cox, H.A.O. Hill, V.J. Lowe, B.N. Oliver, J. Electroanal. Chem. 217 (1987) 331.

[13] M.A. Harmer, H.A.O. Hill, J. Electroanal. Chem. 189 (1985) 229

[14] F.A. Armstrong, H.A.O. Hill, B.N. Oliver, J. Chem. Soc., Chem. Commun. (1984) 976.

[15] F.A. Armstrong, H.A.O. Hill, B.N. Oliver, N.J. Walton, J. Am. Chem. Soc. 106 (1984) 921.

[16] F.A. Armstrong, A.M. Lannon, J. Am. Chem. Soc. 109 (1987) 7211.

[17] D. Datta, H.A.O. Hill, H. Nakayama, J. Electroanal. Chem. 324 (1992) 307.
[18] J.J. Davis, H.A.O. Hill, A.M. Bond, Coord. Chem. Rev. 200-202 (2000) 411.

[19] F.A. Armstrong, A.M. Bond, H.A.O. Hill, I.S.M. Psalti, C.G. Zoski, J. Phys. Chem. 93 (1989) 6485.

[20] F.A. Armstrong, A.M. Bond, H.A.O. Hill, B.N. Oliver, I.S.M. Psalti, J. Am. Chem. Soc. 111 (1989) 9185.

[21] M.M.C. Santos, P.M.P. Sousa, M.L.S. Gonçalves, H. Lopes, I. Moura, J.J.G. Moura, J. Electroanal. Chem. 464 (1999) 76.

[22] M.M.C. Santos, P.M.P. Sousa, M.L.S. Gonçalves, C. Ascenso, I. Moura, J.J.G. Moura, J. Electroanal. Chem. 501 (2001) 173.

[23] J. Haladjian, P. Bianco, F. Nunzi, M. Bruschi, Anal. Chim. Acta 289 (1994) 15.

[24] J. Haladjian, I. Thierry-Chef, P. Bianco, Talanta 43 (1996) 1125.

[25] É. Lojou, P. Bianco, J. Electroanal. Chem. 485 (2000) 71.

[26] M. Bruschi, J. LeGall, Biochim. Biophys. Acta 271 (1972) 279.

[27] T. Yagi, Methods in Enzymology, vol. 243 (Ch. 8), Academic Press, New York, 1994.

[28] L.M. Saraiva, G. Fauque, S. Besson, I. Moura, Eur. J. Biochem. 224 (1994) 1011.

[29] G. Fauque, J.J.G. Moura, S. Besson, L.M. Saraiva, I. Moura, Oceánis 18 (1992) 211

[30] P.N. Palma, L. Krippahl, J.E. Wampler, J.J.G. Moura, Protein Sci. 39 (2000) 372 (http://www.dq.unl.pt/bioin/chemera).

[31] A.J. Bard, L.R. Faulkner, Electrochemical Methods, Fundamentals and Applications, 2nd ed, Wiley, New York, 2001.

[32] E. Laviron, J. Electroanal. Chem. 101 (1979) 19.

[33] K. Aoki, K. Maeda, J. Osteryoung, J. Electroanal. Chem. 272 (1989) 17

[34] P. Bianco, J. Haladjin, Electrochim. Acta 39 (1994) 911

[35] T.L. Poulos, J. Kraut, J. Biol. Chem. 255 (1980) 10322.

[36] F. Millett, C. de Jong, L. Paulson, R.A. Capaldi, Biochemistry 22 (1983) 546. 